\title{
Analysis of Different Stenotic Geometries on Two-Phase Blood Flow
}

\author{
Ramzy M. Abumandour ${ }^{1}$, I. M. Eldesoky ${ }^{1,2}$, A. S. Dawood ${ }^{1}$, M. H. Kamel ${ }^{3,4}$, Samy M. El- \\ Behery $^{5}$ \\ ${ }^{1}$ Department of Basic Engineering Science, Faculty of Engineering, Menoufia University, Shebin El- \\ Kom, Egypt \\ ${ }^{2}$ Department of Basic Engineering Science, Faculty of Engineering, Elmenofia Higher Institute of \\ Engineering and Technology, Elbagor, Egypt \\ ${ }^{3}$ Engineering Mathematics and Physics Department, Faculty of Engineering, Cairo University, Giza, \\ Egypt \\ ${ }^{4}$ Dean of El-Madina Higher Institute of Engineering and Technology, Giza, Egypt \\ ${ }^{5}$ Department of Mechanical Power Engineering, Faculty of Engineering, Menoufia University, Shebin \\ El-Kom, Egypt \\ (Corresponding author: ramzy-0000@yahoo.com )
}

\begin{abstract}
There are many different mathematical models and solution techniques dealing with the blood flows. A twophase (liquid-solid) model solved by a perturbation method will be introduced. Effects of hematocrit, Reynolds number, area reduction, and different geometries of the stenotic channel are studied on the velocity, pressure gradient, and streamlines. The flow characteristics (velocity and pressure) give a great increase as the stenosis classified as a sever stenosis. It is found that, the stenosis geometry has a great effect on the pressure gradient in the axial direction, and also there is a change in velocity profile in each cross section of the geometry. The hematocrit has a noticed effect on the flow velocity and pressure gradient, in which as the hematocrit increases the maximum velocity decreases before the throat while it increased after that section.
\end{abstract}

Keyword: Stenotic geometry; Two-Phase-Flow; incompressible flow; No-slip conditions

\section{Introduction}

Stenotic geometry in the blood flow is one of the important sections in the human arteries. Understanding the behavior of blood flows in this section is necessary because it is playing a role in causing a die from heart attacks. Atherosclerosis is a disease causing the heart attacks as referred to Refs. [1] and [2], This known disease is formed by accumulation of the deposit on the artery walls, the plaque in the artery is known as a stenotic. The stenosis is classified as a mild, moderate, and severe based on the area reduction of the original blood vessel as mentioned in [3]. When the area reduction is less than 54\% the stenosis is classified as a mild, while the moderate stenosis has on area reduction between $54 \%$ and $75 \%$. For the area reduction greater than $75 \%$, this narrowing classified as severe stenosis.

The blood flow through living organisms can be modeled with different mathematical models as a single phase with a Newtonian or non-Newtonian model and two phases with Eulerian-Eulerian or Eulerian-Lagrangian approaches.

The stenosis geometry and area reduction affect the fluid characteristics as, velocity, pressure gradient, wall shear stress, impedance, and separation of the flow. Many authors studied the flow through stenosis with different type (mild, moderate and sever) by many ways (experimentally, analytically and numerically), although the most experimental results used a glycerol-water solution to give a features near to the blood. There are no experimental results dealing with the blood as a two-phase model (plasma and red blood cells) in stenosis artery.

Studying the blood flow through a stenosis was the objective of many authors as [2] and [4-13] attempting to understand the harmful effect on the human. Also, a review article [14] gives more attention on the flow through stenosis. The perturbation analytical solution technique had been used in many researches as [4], [11] and [15-20] while there are many different ways of solution as the 
long wave a proximation as shown by [5-7], [12] and [21-23] and integral method introduced [24].

The mathematical models of this type of flow has a variety of explanation, first the flow was assumed as a single incompressible fluid model with a) Newtonian fluid as assumed in [4-6], [15], [16], [20] and [24] b) Non-Newtonian fluid as [7-11] and [18] and others. Second the flow had been assumed as two fluid layers as a peripheral layer with plasma only and a core region with plasma and red blood cells.

The two fluid model had been studied with Newtonian and Newtonian for each layer by [2] and there is a work dealing with the peripheral layer as a Newtonian while the core layer as a Non-Newtonian [17] and [19].

The most previous works considered the blood as a single phase (fluid) while there were researches using a two phase model as the plasma as a fluid phase and the red blood cells as a particle phase as referred by [21] and [22]. Chakraborty et al. [25] gave a solution for the same model and the same solution technique as in [21] with the stenosis in an inclined artery and included the slip boundary conditions.

Charya [26] introduced a solution for a dusty fluid (two-phase flow) built on the perturbation techniques introduced by Chow and Soda in [15] and [16]. A pulsatile flow had been studied in this flow without including the pressure force in the particles governing equations also the second order of the perturbation not included.

Sankar et al. [27] used the long wave approximation to solve a model with two fluid layers as a peripheral layer treated as a Newtonian fluid while the core region is treated as a two phase (red blood cell suspended in the plasma).

The flow characteristics had been studied by all the previous authors. The impedance, wall shear stress and the separation and reattachment points on the stenotic geometry had been studied under the different mathematical models introduced above.

The aim of this work is to study the effect of different hematocrit (red blood cells concentration in blood), Reynolds number, the area reduction and different geometries of the stenosis on blood flow. Based on the work of Chow and Soda [15], [16] and the work of Charya [26] with using the perturbation method tile the second order system the velocity, pressure gradient, and stream functions will be introduced for different characteristics.

\section{Mathematical Model} quationsE overningG The

The blood flow in a two-dimensional channel is assumed as incompressible, steady, Newtonian, twophase flow as the first phase is the plasma of the blood which will be treated as a fluid phase while the red blood cells are assumed a fixed non deformable solid spheres and treated as a particular phase.

The governing equations for the blood moving through a channel with the previous assumptions are as follows:

$\mathrm{x}$-momentum equation for the fluid phase

$$
\begin{aligned}
& (1-c) \rho_{f}\left(u_{f} \frac{\partial u_{f}}{\partial x}+v_{f} \frac{\partial u_{f}}{\partial y}\right)=-(1-c) \frac{\partial p}{\partial x}+ \\
& (1-c) \mu_{s}\left(\frac{\partial^{2} u_{f}}{\partial x^{2}}+\frac{\partial^{2} u_{f}}{\partial y^{2}}\right)+c S\left(u_{p}-u_{f}\right),
\end{aligned}
$$

$\mathrm{y}$-momentum equation for the fluid phase

$$
\begin{aligned}
& (1-c) \rho_{f}\left(u_{f} \frac{\partial v_{f}}{\partial x}+v_{f} \frac{\partial v_{f}}{\partial y}\right)=-(1-c) \frac{\partial p}{\partial y}+ \\
& (1-c) \mu_{s}\left(\frac{\partial^{2} v_{f}}{\partial x^{2}}+\frac{\partial^{2} v_{f}}{\partial y^{2}}\right)+c S\left(v_{p}-v_{f}\right),
\end{aligned}
$$

Fluid continuity equation

$\frac{\partial}{\partial x}(1-c) u_{f}+\frac{\partial}{\partial y}(1-c) v_{f}=0$,

$\mathrm{x}$-momentum equation for the particle phase

$c \rho_{p}\left(u_{p} \frac{\partial u_{p}}{\partial x}+v_{p} \frac{\partial u_{p}}{\partial y}\right)=-c \frac{\partial p}{\partial x}+c S\left(u_{f}-u_{p}\right)$

$\mathrm{y}$-momentum equation for the particle phase

$c \rho_{p}\left(u_{p} \frac{\partial v_{p}}{\partial x}+v_{p} \frac{\partial v_{p}}{\partial y}\right)=-c \frac{\partial p}{\partial y}+c S\left(v_{f}-v_{p}\right)$

Particle continuity equation

$\frac{\partial}{\partial x} c u_{p}+\frac{\partial}{\partial y} c v_{p}=0$,

where $u_{f}, v_{f}$ are the $(x, y)$ fluid velocity components, $u_{p}, v_{p}$ are the $(x, y)$ particles velocity components, $c$ is the red blood cells volume concentration (hematocrit) and is assumed a constant in all the domain, $S$ is the drag coefficient, and $\mu_{s}$ is the suspension viscosity.

The suspension viscosity can be defined as [28] and [29].

$\mu_{s}=\mu_{f} * \frac{1}{1-p c}$

$p=0.07 e^{\left(2.49 c+\frac{1107}{T} e^{-1.69 c}\right)}$,

where $\mathrm{T}$ is the absolute temperature measured by Kelvin.

The geometry of the test section for narrowing the artery is based on the equation

$\eta(x)=y_{\text {wall }}=H-h(x)$,

where $H$ is the artery height without narrowing and $h(x)$ is the amount of area reduction at every axial distance.

The geometry parameters are shown in Fig. 1.

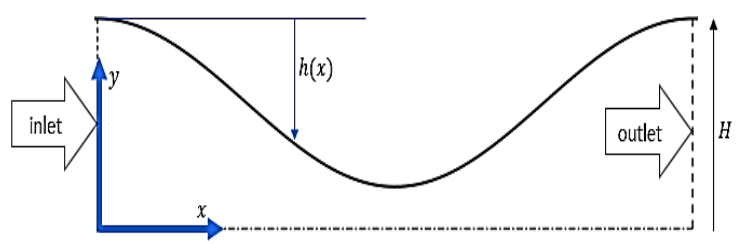

Figure.1- The test section geometry 


\section{Boundary Conditions}

For this mathematical model no slip velocity near the walls $\left[u_{f}(x, \eta)=0, v_{f}(x, \eta)=0\right]$ and for the symmetry axis the velocity will be maximum as $\frac{\partial u_{f}(x, 0)}{\partial y}=0$. While the normalized flow rate through the domain will be $\int_{0}^{\eta} u_{f} d y=1$

For this model, the particles velocity at boundary is not necessary because it included in the flow rate calculations.

\section{Dimensionless Analysis}

From the geometry of the stenosis the dimensionless parameters are shown below based on Chow and Soda [16]

$$
\begin{gathered}
x^{*}=\frac{x}{L}, y^{*}=\frac{y}{H}, u_{f}^{*}=\frac{u_{f}}{u_{o}}, u_{p}^{*}=\frac{u_{p}}{u_{o}}, v_{f}^{*}=\frac{L v_{f}}{H u_{o}}, \\
v_{p}^{*}=\frac{L v_{p}}{H u_{o}}, \quad p^{*}=\frac{H^{2} p}{u_{o} \mu_{f} L}
\end{gathered}
$$

The dimensionless numbers used in this analysis will be $\left(R e=\rho_{f} u_{o} H / \mu_{f}\right)$ the Reynolds number,

( $\delta=H / L)$ the perturbation parameter, the ratio of the viscosity $\left(\mu^{\prime}=\mu_{s} / \mu_{f}\right)$, the density ratio

$\left(D=\rho_{p} / \rho_{f}\right)$ and the suspension parameter is $\left(M=\frac{S H^{2}}{(1-c) \mu_{f}}\right)$

After applying the dimensionless parameters and dropping the stars, then the system (1) - (6) and its boundary conditions now become,

$$
\begin{aligned}
& \operatorname{Re} \delta\left(u_{f} \frac{\partial u_{f}}{\partial x}+v_{f} \frac{\partial u_{f}}{\partial y}\right)=-\frac{\partial p}{\partial x}+\mu^{\prime}\left(\delta^{2} \frac{\partial^{2} u_{f}}{\partial x^{2}}+\frac{\partial^{2} u_{f}}{\partial y^{2}}\right) \\
& +c M\left(u_{p}-u_{f}\right), \\
& \operatorname{Re} \delta^{3}\left(u_{f} \frac{\partial v_{f}}{\partial x}+v_{f} \frac{\partial v_{f}}{\partial y}\right)=\mu^{\prime}\left(\delta^{4} \frac{\partial^{2} v_{f}}{\partial x^{2}}+\delta^{2} \frac{\partial^{2} v_{f}}{\partial y^{2}}\right) \\
& -\partial p / \partial y+c M \delta^{2}\left(v_{p}-v_{f}\right), \\
& \frac{\partial u_{f}}{\partial x}+\frac{\partial v_{f}}{\partial y}=0 \\
& D \operatorname{Re} \delta\left(u_{p} \frac{\partial u_{p}}{\partial x}+v_{p} \frac{\partial u_{p}}{\partial y}\right)=(1-c) M\left(u_{f}-u_{p}\right) \\
& -\partial p / \partial x \\
& D \operatorname{Re} \delta^{3}\left(u_{p} \frac{\partial v_{p}}{\partial x}+v_{p} \frac{\partial v_{p}}{\partial y}\right)=(1-c) M \delta^{2}\left(v_{f}-v_{p}\right) \\
& -\partial p / \partial y \\
& \frac{\partial u_{p}}{\partial x}+\frac{\partial v_{p}}{\partial y}=0 .
\end{aligned}
$$

\section{Method of Solution}

Assuming the ratio $\delta$ between the height to the stenosis length to be small parameter, this small parameter is called the perturbation parameter, we obtain the solution for the system of equation as a power series in terms of $\delta$, by expanding $u_{f}, v_{f} u_{p}$, $v_{p}, p$ as follows:

The small parameter is called the perturbation parameter

$$
\begin{aligned}
& u_{f}=u_{f o}+\delta u_{f 1}+\delta^{2} u_{f 2}+\delta^{3} u_{f 3}+\cdots \\
& v_{f}=v_{f o}+\delta v_{f 1}+\delta^{2} v_{f 2}+\delta^{3} v_{f 3}+\cdots \\
& u_{p}=u_{p o}+\delta u_{p 1}+\delta^{2} u_{p 2}+\delta^{3} u_{p 3}+\cdots \\
& v_{p}=v_{p o}+\delta v_{p 1}+\delta^{2} v_{p 2}+\delta^{3} v_{p 3}+\cdots \\
& p=p_{o}+\delta p_{1}+\delta^{2} p_{2}+\delta^{3} p_{3}+\cdots
\end{aligned}
$$

From this procedure substituting equation (15) in the system of equations (9)-(14), and collecting like powers terms of $\delta$, we obtain three-sets of partial differential equations together with their boundary conditions.

Zero-set system

$$
\begin{aligned}
& 0=-\frac{\partial p_{o}}{\partial x}+\mu^{\prime}\left(\frac{\partial^{2} u_{f o}}{\partial y^{2}}\right)+c M\left(u_{p o}-u_{f o}\right), \\
& 0=-(1-c) \frac{\partial p_{o}}{\partial y}, \\
& \frac{\partial u_{f o}}{\partial x}+\frac{\partial v_{f o}}{\partial y}=0, \\
& 0=-c \frac{\partial p_{o}}{\partial x}+c(1-c) M\left(u_{f o}-u_{p o}\right), \\
& 0=-c \frac{\partial p_{o}}{\partial y}, \\
& \frac{\partial u_{p o}}{\partial x}+\frac{\partial v_{p o}}{\partial y}=0
\end{aligned}
$$

With boundary conditions for the zero-set system

$u_{f o}(x, \eta)=0$,

$v_{f o}(x, \eta)=0$,

$\frac{\partial u_{f o}(x, 0)}{\partial y}=0$,

$\int_{0}^{\eta} u_{f o} d y=1$.

First-set system

$$
\begin{aligned}
& \operatorname{Re}\left(u_{f o} \frac{\partial u_{f o}}{\partial x}+v_{f o} \frac{\partial u_{f o}}{\partial y}\right)=-\frac{\partial p_{1}}{\partial x}+\mu^{\prime}\left(\frac{\partial^{2} u_{f 1}}{\partial y^{2}}\right) \\
& +c M\left(u_{p 1}-u_{f 1}\right) \\
& 0=-(1-c) \frac{\partial p_{1}}{\partial y}, \\
& \frac{\partial u_{f 1}}{\partial x}+\frac{\partial v_{f 1}}{\partial y}=0, \\
& D R e\left(u_{p o} \frac{\partial u_{p o}}{\partial x}+v_{p o} \frac{\partial u_{p o}}{\partial y}\right)=-\frac{\partial p_{1}}{\partial x}+(1-c) \\
& M\left(u_{f 1}-u_{p 1}\right), \\
& 0=-c \frac{\partial p_{1}}{\partial y}, \\
& \frac{\partial u_{p 1}}{\partial x}+\frac{\partial v_{p 1}}{\partial y}=0 .
\end{aligned}
$$

With boundary conditions for the first-set system

$$
\begin{aligned}
& u_{f 1}(x, \eta)=0, \\
& v_{f 1}(x, \eta)=0, \\
& \frac{\partial u_{f 1}(x, 0)}{\partial y}=0, \\
& \int_{0}^{\eta} u_{f 1} d y=0 .
\end{aligned}
$$




$$
\begin{aligned}
& \text { Second-set system } \\
& \operatorname{Re}\left(u_{f o} \frac{\partial u_{f 1}}{\partial x}+u_{f 1} \frac{\partial u_{f o}}{\partial x}+v_{f o} \frac{\partial u_{f 1}}{\partial y}+v_{f 1} \frac{\partial u_{f o}}{\partial y}\right) \\
& =-\frac{\partial p_{2}}{\partial x}+\mu^{\prime}\left(\frac{\partial^{2} u_{f o}}{\partial x^{2}}+\frac{\partial^{2} u_{f 2}}{\partial y^{2}}\right)+c M\left(u_{p 2}-u_{f 2}\right) \\
& 0=-\frac{\partial p_{2}}{\partial y}+\mu^{\prime} \frac{\partial^{2} v_{o}}{\partial y^{2}}+c M\left(v_{p o}-v_{f o}\right) \\
& \frac{\partial u_{f 2}}{\partial x}+\frac{\partial v_{f 2}}{\partial y}=0 \\
& D R e\left(u_{p o} \frac{\partial u_{p 1}}{\partial x}+u_{p 1} \frac{\partial u_{p o}}{\partial x}+v_{p o} \frac{\partial u_{p 1}}{\partial y}+v_{p 1} \frac{\partial u_{p o}}{\partial y}\right)= \\
& -\frac{\partial p_{2}}{\partial x}+(1-c) M\left(u_{f 2}-u_{p 2}\right) \\
& 0=-c \frac{\partial p_{2}}{\partial y}+c(1-c) M\left(v_{f o}-v_{p o}\right) \\
& \frac{\partial u_{p 2}}{\partial x}+\frac{\partial v_{p 2}}{\partial y}=0 .
\end{aligned}
$$

With boundary conditions for the second-set system

$u_{f 2}(x, \eta)=0$,

$v_{f 2}(x, \eta)=0$,

$\frac{\partial u_{f 2}(x, 0)}{\partial y}=0$,

$\int_{0}^{\eta} u_{f 2} d y=0$.

Now, our main concern is to discover the solution of the above differential equations, for $u_{(f, p)_{o}}, v_{(f, p)_{o}}$, $u_{(f, p)_{1}}, v_{(f, p)_{1}}, u_{(f, p)_{2}}, v_{(f, p)_{2}}$.

Zero-order solution is:

$u_{f o}=\frac{1}{\eta}\left(-\frac{3}{2} \zeta^{2}+\frac{3}{2}\right)$,

$v_{f o}=\frac{\eta^{\prime}}{\eta}\left(-\frac{3}{2} \zeta^{3}+\frac{3}{2} \zeta\right)$,

$\psi_{f o}=-\frac{1}{2} \zeta^{3}+\frac{3}{2} \zeta$,

$\frac{\partial p_{o}}{\partial x}=(1-c) \mu^{\prime}\left(\frac{1}{\eta^{3}}(-3)\right)$.

(49)

$$
\begin{aligned}
& u_{p o}=u_{f o}+\frac{\mu^{\prime}}{M}\left(\frac{1}{\eta^{3}}(3)\right), \\
& v_{p o}=v_{f o}+\frac{\mu^{\prime}}{M}\left(\frac{\eta^{\prime}}{\eta^{3}}(9 \zeta)\right) .
\end{aligned}
$$

First-order solution is:

$$
\begin{aligned}
& u_{f 1}=\frac{(1-c+c D)}{1-c} \frac{R e}{\mu^{\prime}}\left(\frac{\eta^{\prime}}{\eta}\left(\begin{array}{c}
-\frac{3}{40} \zeta^{6}+\frac{3}{8} \zeta^{4} \\
-\frac{99}{280} \zeta^{2}+\frac{3}{56}
\end{array}\right)\right), \\
& v_{f 1}=\frac{(1-c+c D)}{1-c} \frac{R e}{\mu^{\prime}}\left(\begin{array}{c}
\frac{\eta^{\prime 2}}{\eta}\left(\begin{array}{c}
-\frac{3}{40} \zeta^{7}+\frac{3}{8} \zeta^{5} \\
-\frac{99}{280} \zeta^{3}+\frac{3}{56} \zeta
\end{array}\right) \\
+\eta^{\prime \prime}\left(\begin{array}{c}
\frac{3}{280} \zeta^{7}-\frac{3}{40} \zeta^{5} \\
+\frac{33}{280} \zeta^{3}-\frac{3}{56} \zeta
\end{array}\right)
\end{array}\right) \text {, } \\
& \psi_{f 1}=\frac{(1-c+c D)}{1-c} \frac{R e}{\mu^{\prime}}\left(\eta^{\prime}\left(\begin{array}{c}
-\frac{3}{280} \zeta^{7}+\frac{3}{40} \zeta^{5} \\
-\frac{33}{280} \zeta^{3}+\frac{3}{56} \zeta
\end{array}\right)\right) \text {, }
\end{aligned}
$$

$$
\begin{aligned}
& \frac{\partial p_{1}}{\partial x}=\operatorname{Re}\left(\begin{array}{c}
(1-c+c D)\left(\frac{\eta^{\prime}}{\eta^{3}}\left(\frac{54}{35}\right)\right) \\
c D\left(\begin{array}{c}
\frac{\mu^{\prime}}{M}\left(\frac{\eta^{\prime}}{\eta^{5}}(18)\right) \\
+\left(\frac{\mu^{\prime}}{M}\right)^{2}\left(\frac{\eta^{\prime}}{\eta^{7}}(27)\right)
\end{array}\right)
\end{array}\right) \text {. } \\
& u_{p 1}=u_{f 1}+\frac{R e}{M}\left(\begin{array}{c}
\frac{D}{1-c}\left(\frac{\eta^{\prime}}{\eta^{3}}\left(\frac{9}{4} \zeta^{4}-\frac{9}{2} \zeta^{2}+\frac{9}{4}\right)\right) \\
+\frac{(1-c+c D)}{1-c}\left(\frac{\eta^{\prime}}{\eta^{3}}\left(-\frac{54}{35}\right)\right) \\
+D\left(\begin{array}{c}
\frac{\mu^{\prime}}{M}\left(\frac{\eta^{\prime}}{\eta^{5}}(18)\right)+ \\
\left(\frac{\mu^{\prime}}{M}\right)^{2}\left(\frac{\eta^{\prime}}{\eta^{7}}(27)\right)
\end{array}\right)
\end{array}\right) \\
& v_{p 1}=v_{f 1}+\frac{R e}{M}\left(\begin{array}{c}
\frac{D}{1-c}\left(\begin{array}{c}
\frac{\eta^{\prime 2}}{\eta^{3}}\left(\frac{63}{20} \zeta^{5}-\frac{15}{2} \zeta^{3}+\frac{27}{4} \zeta\right) \\
+\frac{\eta^{\prime \prime}}{\eta^{2}}\left(-\frac{9}{20} \zeta^{5}+\frac{3}{2} \zeta^{3}-\frac{9}{4} \zeta\right)
\end{array}\right) \\
+\frac{(1-c+c D)}{1-c}\left(\frac{\eta \eta^{\prime \prime}-3 \eta^{\prime 2}}{\eta^{3}}\left(\frac{54}{35} \zeta\right)\right) \\
+D\left(\begin{array}{c}
\frac{\mu^{\prime}}{M}\left(\frac{\eta \eta^{\prime \prime}-5 \eta^{\prime 2}}{\eta^{5}}(-18 \zeta)\right)+ \\
\left(\frac{\mu^{\prime}}{M}\right)^{2}\left(\frac{\eta \eta^{\prime \prime}-7 \eta^{\prime 2}}{\eta^{7}}(-27 \zeta)\right)
\end{array}\right)
\end{array}\right)
\end{aligned}
$$

\section{Second-order solution is:}

$$
\begin{aligned}
& u_{f 2}=A_{1}+\frac{(1-c+c D)}{(1-c)^{2}} \frac{R e^{2}}{\mu^{\prime 2}}\left(\begin{array}{c}
c D Q_{1} \eta A_{3}+ \\
(1-c+c D) A_{2}
\end{array}\right) \\
& +\frac{c D^{2}}{(1-c)^{2}} \frac{R e^{2}}{\mu^{\prime}}\left(A_{4}(1-c)+Q_{1} A_{5}\right) \\
& v_{f 2}=E_{1}+\frac{(1-C+C D)}{(1-C)^{2}} \frac{R e^{2}}{\mu^{\prime 2}}\left(\begin{array}{c}
(1-C+C D) E_{2} \\
-C D Q_{1} E_{3}-C D Q_{1}^{\prime} E_{4}
\end{array}\right) \\
& +\frac{C D^{2}}{(1-C)^{2}} \frac{R e^{2}}{\mu^{\prime}}\left(\begin{array}{c}
Q_{1} E_{5}+Q_{1}^{\prime} E_{6}+Q_{1}^{2} E_{7}+Q_{1} Q_{1}^{\prime} E_{8} \\
-(1-c)\left(\left(Q_{2} E_{9}+Q_{2}^{\prime \prime} E_{10}+Q_{2}^{\prime} E_{11}\right)\right.
\end{array}\right)
\end{aligned}
$$

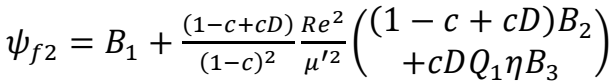

$$
\begin{aligned}
& +\frac{c D^{2}}{(1-c)^{2}} \frac{R e^{2}}{\mu^{\prime}}\left(Q_{1} B_{4}+(1-c) B_{5}\right) \\
& \frac{\partial p_{2}}{\partial x}=(1-c) \mu^{\prime} F_{1}+\frac{(1-c+c D)}{1-c} \frac{R e^{2}}{\mu^{\prime}}\left(\begin{array}{c}
(1-c+c D) F_{2} \\
+c D Q_{1} \eta F_{3}
\end{array}\right) \\
& +\frac{c D^{2}}{1-c} R e^{2}\left(Q_{1} F_{5}+(1-c) F_{4}\right) \\
& u_{p 2}=u_{f 2}-\frac{(1-c+c D)}{(1-c)^{2}} \frac{R e^{2}}{M \mu^{\prime}}\left(\begin{array}{c}
(1-c+c D) G_{2} \\
+D\left(G_{3}+Q_{1} G_{5}\right)
\end{array}\right) \\
& -\frac{D^{2} R e^{2}}{(1-c)^{2} M}\left((1-c) G_{4}+G_{6}\right)+\frac{(2-c) \mu^{\prime} G_{1}}{c M} \\
& v_{p 2}=v_{f 2}+\frac{(1-c+c D)}{(1-c)^{2}} \frac{R e^{2}}{M \mu^{\prime}}\left(\begin{array}{c}
(1-c+c D) H_{6} \\
+D Q_{1} H_{3} \\
+D\left(H_{2}+H_{4}+H_{5}\right)
\end{array}\right)
\end{aligned}
$$



Different Stenotic Geometries on Two-Phase Blood Flow"

$\frac{D^{2}}{(1-c)^{2}} \frac{R e^{2}}{M}\left(\begin{array}{c}(1-c)\left(\begin{array}{c}H_{7}+H_{8} \\ -(1-c) H_{9}\end{array}\right)+ \\ Q_{1} H_{10}+Q_{1}^{\prime} H_{11} \\ +Q_{1}^{2} H_{12}+Q_{1} Q_{1}^{\prime} H_{13}\end{array}\right)+\frac{2-c}{c} \frac{\mu^{\prime}}{M} H_{1}$

Where

$A_{1}=\left(\begin{array}{c}\frac{\eta^{\prime 2}}{\eta}\left(3 \zeta^{4}-\frac{18}{5} \zeta^{2}+\frac{3}{5}\right)+ \\ \eta^{\prime \prime}\left(-\frac{3}{4} \zeta^{4}+\frac{9}{10} \zeta^{2}-\frac{3}{20}\right)\end{array}\right)$

$A_{2}=\left(\begin{array}{c}\frac{{\eta^{\prime}}^{2}}{\eta}\left(\begin{array}{c}-\frac{1}{400} \zeta^{10}+\frac{27}{1120} \zeta^{8}-\frac{51}{700} \zeta^{6} \\ +\frac{57}{560} \zeta^{4}-\frac{24666}{431200} \zeta^{2}+\frac{115}{17248}\end{array}\right)+ \\ \eta^{\prime \prime}\left(\begin{array}{l}\frac{1}{1120} \zeta^{10}-\frac{9}{1120} \zeta^{8}+\frac{69}{2800} \zeta^{6} \\ -\frac{3}{80} \zeta^{4}+\frac{9837}{431200} \zeta^{2}-\frac{1213}{431200}\end{array}\right)\end{array}\right)$

$A_{3}=\left(\begin{array}{c}\frac{\eta^{\prime 2}}{\eta}\left(\begin{array}{c}-\frac{3}{280} \zeta^{8}+\frac{1}{20} \zeta^{6} \\ -\frac{1}{20} \zeta^{2}+\frac{3}{280}\end{array}\right) \\ \eta^{\prime \prime}\left(\begin{array}{c}\mu^{\prime}\left(-\frac{9}{140} \zeta^{4}+\frac{27}{350} \zeta^{2}-\frac{9}{700}\right) \\ -\frac{3}{2240} \zeta^{8}+\frac{1}{80} \zeta^{6}-\frac{33}{1120} \zeta^{4} \\ +\frac{59}{2800} \zeta^{2}-\frac{31}{11200}\end{array}\right)\end{array}\right)$

$A_{4}=\left(Q_{2}^{\prime} \eta+3 \eta^{\prime} Q_{2}\right)\left(\frac{1}{8} \zeta^{4}-\frac{3}{20} \zeta^{2}+\frac{1}{40}\right)$

$A_{5}=\left(\begin{array}{c}\eta^{\prime 2}\left(\begin{array}{c}\frac{9}{140} \zeta^{8}-\frac{7}{20} \zeta^{6}+\frac{3}{4} \zeta^{4} \\ -\frac{15}{28} \zeta^{2}+\frac{1}{14}+ \\ Q_{1} \eta\left(\begin{array}{c}\frac{1}{20} \zeta^{6}+\frac{1}{4} \zeta^{4} \\ -\frac{51}{140} \zeta^{2}+\frac{9}{140}\end{array}\right)\end{array}\right) \\ +\eta \eta^{\prime \prime}\left(\begin{array}{c}-\frac{27}{2240} \zeta^{8}+\frac{1}{16} \zeta^{6}-\frac{3}{32} \zeta^{4} \\ +\frac{27}{560} \zeta^{2}-\frac{11}{2240}+ \\ Q_{1} \eta\left(\begin{array}{c}\frac{1}{40} \zeta^{6}-\frac{1}{8} \zeta^{4} \\ +\frac{33}{280} \zeta^{2}-\frac{1}{56}\end{array}\right)\end{array}\right)\end{array}\right)$

$B_{1}=\left(\begin{array}{c}\eta^{\prime 2}\left(\frac{3}{5} \zeta^{5}-\frac{6}{5} \zeta^{3}+\frac{3}{5} \zeta\right)+ \\ \eta \eta^{\prime \prime}\left(-\frac{3}{20} \zeta^{5}+\frac{3}{10} \zeta^{3}-\frac{3}{20} \zeta\right)\end{array}\right)$

$B_{2}=\left(\begin{array}{c}\eta^{\prime 2}\left(\begin{array}{c}-\frac{1}{4400} \zeta^{11}+\frac{3}{1120} \zeta^{9}-\frac{51}{4900} \zeta^{7} \\ +\frac{57}{2800} \zeta^{5}-\frac{8222}{431200} \zeta^{3}+\frac{115}{17248} \zeta\end{array}\right) \\ +\eta \eta^{\prime \prime}\left(\begin{array}{c}\frac{1}{12320} \zeta^{11}-\frac{1}{1120} \zeta^{9}+\frac{69}{19600} \zeta^{7} \\ -\frac{3}{400} \zeta^{5}+\frac{3279}{431200} \zeta^{3}-\frac{1213}{431200} \zeta\end{array}\right)\end{array}\right)$
$\left.B_{3}=\left(\begin{array}{c}\eta^{\prime 2}\left(\begin{array}{c}-\frac{1}{840} \zeta^{9}+\frac{1}{140} \zeta^{7} \\ -\frac{1}{60} \zeta^{3}+\frac{3}{280} \zeta\end{array}\right) \\ \eta \eta^{\prime \prime}\left(\begin{array}{c}\mu^{\prime}\left(-\frac{9}{700} \zeta^{5}+\frac{9}{350} \zeta^{3}-\frac{9}{700} \zeta\right) \\ -\frac{1}{6720} \zeta^{9}+\frac{1}{560} \zeta^{7} \\ -\frac{33}{5600} \zeta^{5}+\frac{59}{8400} \zeta^{3}-\frac{31}{11200} \zeta\end{array}\right)\end{array}\right)\right)$

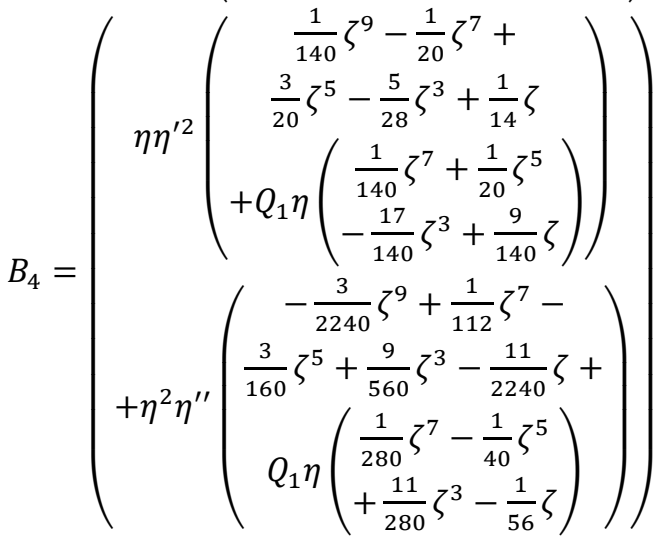

$B_{5}=\left(Q_{2}^{\prime} \eta^{2}+3 \eta \eta^{\prime} Q_{2}\right)\left(\frac{1}{40} \zeta^{5}-\frac{1}{20} \zeta^{3}+\frac{1}{40} \zeta\right)$

$E_{1}=\left(\begin{array}{c}\frac{\eta^{\prime 3}}{\eta}\left(3 \zeta^{5}-\frac{18}{5} \zeta^{3}+\frac{3}{5} \zeta\right) \\ +\eta^{\prime \prime} \eta^{\prime}\left(\frac{3}{10} \zeta^{5}-\frac{6}{5} \zeta^{3}+\frac{9}{10} \zeta\right) \\ -\eta \eta^{\prime \prime \prime}\left(\frac{-3}{20} \zeta^{5}+\frac{3}{10} \zeta^{3}-\frac{3}{20} \zeta\right)\end{array}\right)$

$E_{2}=\left(\begin{array}{c}\frac{\eta^{\prime 3}}{\eta}\left(\begin{array}{c}\frac{-1}{400} \zeta^{11}+\frac{27}{1120} \zeta^{9}-\frac{51}{700} \zeta^{7} \\ +\frac{57}{560} \zeta^{5}-\frac{24666}{431200} \zeta^{3}+\frac{115}{17248} \zeta\end{array}\right) \\ +\eta^{\prime \prime} \eta^{\prime}\left(\begin{array}{c}\frac{-39}{30800} \zeta^{11}+\frac{1}{70} \zeta^{9}-\frac{411}{9800} \zeta^{7} \\ +\frac{99}{1400} \zeta^{5}-\frac{1643}{30800} \zeta^{3}+\frac{23}{43120} \zeta\end{array}\right) \\ +\eta^{\prime \prime \prime} \eta\left(\begin{array}{c}\frac{1}{12320} \zeta^{11}-\frac{1}{1120} \zeta^{9}+\frac{69}{19600} \zeta^{7} \\ -\frac{3}{400} \zeta^{5}+\frac{3279}{431200} \zeta^{3}-\frac{1213}{431200} \zeta\end{array}\right)\end{array}\right)$

$E_{3}=\left(\begin{array}{c}\eta^{3}\left(\frac{1}{105} \zeta^{9}-\frac{3}{70} \zeta^{7}+\frac{1}{30} \zeta^{3}\right)+ \\ \eta \eta^{\prime} \eta^{\prime \prime}\left(\begin{array}{c}\frac{-3}{2240} \zeta^{9}+\frac{3}{560} \zeta^{7}-\frac{33}{800} \zeta^{5} \\ -\frac{113}{2800} \zeta^{3}+\frac{209}{11200} \\ +\mu^{\prime}\left(\frac{27}{700} \zeta^{5}-\frac{9}{350} \zeta^{3}-\frac{9}{700} \zeta\right)\end{array}\right)+ \\ \eta^{2} \eta^{\prime \prime \prime}\left(\begin{array}{c}\mu^{\prime}\left(\frac{-9}{700} \zeta^{5}+\frac{9}{350} \zeta^{3}-\frac{9}{700} \zeta\right)- \\ \frac{1}{6720} \zeta^{9}+\frac{1}{560} \zeta^{7}-\frac{33}{5600} \zeta^{5} \\ +\frac{59}{8400} \zeta^{3}-\frac{31}{11200} \zeta\end{array}\right)\end{array}\right)$ 

Different Stenotic Geometries on Two-Phase Blood Flow"



$$
\begin{aligned}
& E_{5}=\left(\begin{array}{c}
\eta^{\prime 3}\left(\frac{8}{140} \zeta^{9}-\frac{6}{20} \zeta^{7}+\frac{3}{5} \zeta^{5}-\frac{10}{28} \zeta^{3}\right)+ \\
\eta \eta^{\prime} \eta^{\prime \prime}\left(\begin{array}{c}
\frac{-53}{2240} \zeta^{9}+\frac{81}{560} \zeta^{7}- \\
\frac{57}{160} \zeta^{5}+\frac{209}{560} \zeta^{3}-\frac{309}{2240} \zeta
\end{array}\right)+ \\
\eta^{2} \eta^{\prime \prime \prime}\left(\begin{array}{c}
\frac{-3}{2240} \zeta^{9}+\frac{1}{112} \zeta^{7}- \\
\frac{3}{160} \zeta^{5}+\frac{9}{560} \zeta^{3}-\frac{11}{240} \zeta
\end{array}\right)
\end{array}\right) \\
& E_{6}=\left(\begin{array}{c}
\eta \eta^{\prime 2}\left(\begin{array}{c}
\frac{1}{140} \zeta^{9}-\frac{1}{20} \zeta^{7}+ \\
\frac{3}{20} \zeta^{5}-\frac{5}{28} \zeta^{3}+\frac{1}{14} \zeta
\end{array}\right)+ \\
\eta^{2} \eta^{\prime \prime}\left(\begin{array}{c}
\frac{-3}{2240} \zeta^{9}+\frac{1}{112} \zeta^{7}- \\
\frac{3}{160} \zeta^{5}+\frac{9}{560} \zeta^{3}-\frac{11}{2240} \zeta
\end{array}\right)
\end{array}\right) \\
& E_{7}=\left(\begin{array}{c}
\eta \eta^{\prime 3}\left(\frac{1}{28} \zeta^{7}+\frac{3}{20} \zeta^{5}-\frac{17}{70} \zeta^{3}-\frac{9}{140} \zeta\right)+ \\
\eta^{2} \eta^{\prime} \eta^{\prime \prime}\left(\frac{-3}{20} \zeta^{5}+\frac{17}{70} \zeta^{3}-\frac{13}{140} \zeta\right)+ \\
\eta^{3} \eta^{\prime \prime}\left(\frac{1}{280} \zeta^{7}-\frac{1}{40} \zeta^{5}++\frac{11}{280} \zeta^{3}-\frac{1}{56} \zeta\right)
\end{array}\right) \\
& E_{8}=\left(\begin{array}{l}
\eta^{2} \eta^{\prime 2}\left(\frac{1}{70} \zeta^{7}+\frac{1}{10} \zeta^{5}-\frac{17}{70} \zeta^{3}+\frac{9}{70} \zeta\right)+ \\
\eta^{3} \eta^{\prime \prime}\left(\frac{1}{140} \zeta^{7}-\frac{1}{20} \zeta^{5}+\frac{11}{140} \zeta^{3}-\frac{1}{28} \zeta\right)
\end{array}\right) \\
& E_{9}=\left(\begin{array}{c}
\eta^{\prime 2}\left(-\frac{3}{10} \zeta^{5}+\frac{3}{10} \zeta^{3}\right) \\
+\eta \eta^{\prime \prime}\left(\frac{3}{40} \zeta^{5}-\frac{3}{20} \zeta^{3}+\frac{3}{40} \zeta\right)
\end{array}\right) \\
& E_{10}=\eta^{2}\left(\frac{1}{40} \zeta^{5}-\frac{1}{20} \zeta^{3}+\frac{1}{40} \zeta\right) \\
& E_{11}=\eta \eta^{\prime}\left(-\frac{1}{20} \zeta^{3}+\frac{1}{10} \zeta\right) \\
& F_{1}=\left(\frac{\eta^{\prime 2}}{\eta^{3}}\left(-18 \zeta^{2}+\frac{21}{5}\right)+\frac{\eta^{\prime \prime}}{\eta^{2}}\left(\frac{9}{2} \zeta^{2}-\frac{3}{10}\right)\right) \\
& F_{2}=\left(\frac{{\eta^{\prime}}^{2}}{\eta^{3}}\left(\frac{624}{13475}\right)+\frac{\eta^{\prime \prime}}{\eta^{2}}\left(-\frac{468}{13475}\right)\right) \\
& F_{3}=\left(\begin{array}{c}
\frac{\eta^{\prime 2}}{\eta^{3}}\left(\left(\mu^{\prime}\left(\frac{108}{35}\right)+\frac{4}{35}\right)+Q_{1} \eta \mu^{\prime} \frac{108}{35}\right) \\
+\frac{\eta^{\prime \prime}}{\eta^{2}}\left(\left(\mu^{\prime}\left(\frac{162}{175}\right)-\frac{2}{175}\right)+Q_{1} \eta \mu^{\prime}\left(\frac{-54}{105}\right)\right)
\end{array}\right) \\
& F_{4}=\left(\frac{Q_{2}^{\prime}}{\eta}\left(-\frac{9}{5}\right)+\frac{\eta^{\prime} Q_{2}}{\eta^{2}}\left(\frac{3}{5}\right)+Q_{2}^{\prime} Q_{1}-3 \frac{\eta^{\prime} Q_{2} Q_{1}}{\eta}\right) \\
& F_{5}=\left(\begin{array}{c}
\frac{\eta^{\prime 2}}{\eta^{2}}\left(\frac{24}{7}+Q_{1} \eta\left(\frac{132}{35}\right)\right) \\
+\frac{\eta^{\prime \prime}}{\eta}\left(-\frac{36}{35}+Q_{1} \eta\left(-\frac{18}{35}\right)\right)
\end{array}\right) \\
& G_{1}=\left(\frac{\eta^{\prime 2}}{\eta^{3}}\left(-18 \zeta^{2}+\frac{21}{5}\right)+\frac{\eta^{\prime \prime}}{\eta^{2}}\left(\frac{9}{2} \zeta^{2}-\frac{3}{10}\right)\right) \\
& G_{2}=\left(\frac{{\eta^{\prime}}^{2}}{\eta^{3}}\left(\frac{624}{13475}\right)+\frac{\eta^{\prime \prime}}{\eta^{2}}\left(-\frac{468}{13475}\right)\right)
\end{aligned}
$$






$$
\begin{aligned}
& H_{4}=(1-c)\left(\begin{array}{c}
\frac{Q_{1}^{2} \eta^{\prime 3}}{\eta}-2 Q_{1}^{2} \eta^{\prime} \eta^{\prime \prime} \\
+2 Q_{1} Q_{1}^{\prime} \eta^{\prime 2}
\end{array}\right) \mu^{\prime}\left(\frac{108}{35}\right) \zeta \\
& H_{5}=(1-c)\left(\eta \eta^{\prime \prime \prime} Q_{1}^{2}+2 \eta \eta^{\prime \prime} Q_{1}^{\prime} Q_{1}\right) \mu^{\prime}\left(\frac{54}{105}\right) \zeta \\
& H_{6}=\left(\frac{\eta^{33}}{\eta^{3}}\left(\frac{-1872}{13475} \zeta\right)+\frac{\eta^{\prime} \eta^{\prime \prime}}{\eta^{2}}\left(\frac{312}{1925} \zeta\right)+\frac{\eta^{\prime \prime \prime}}{\eta}\left(\frac{-468}{13475} \zeta\right)\right) \\
& H_{7}=\left(\begin{array}{c}
-Q_{2}^{\prime} \frac{\eta^{\prime}}{\eta}\left(\frac{3}{2} \zeta^{3}-\frac{3}{10} \zeta+(1-c) \frac{9}{5} \zeta\right)+ \\
Q_{2}^{\prime \prime}\left(\frac{1}{2} \zeta^{3}-\frac{3}{10} \zeta+(1-c) \frac{9}{5} \zeta\right) \\
-Q_{2} \frac{\eta^{\prime 2}}{\eta^{2}}\left(\frac{9}{2} \zeta^{3}-\frac{9}{10} \zeta+(1-c)\left(-\frac{3}{5} \zeta\right)\right)
\end{array}\right) \\
& H_{8}=\left(Q_{2}\left(\frac{\eta^{\prime \prime}}{\eta}-\frac{\eta^{\prime 2}}{\eta^{2}}\right)+Q_{2}^{\prime} \frac{\eta^{\prime}}{\eta}\right)\left(\begin{array}{c}
\frac{3}{2} \zeta^{3}-\frac{9}{10} \zeta+ \\
(1-c)\left(-\frac{3}{5} \zeta\right)
\end{array}\right) \\
& H_{9}=\left(\begin{array}{c}
Q_{1} Q_{2}^{\prime} \eta^{\prime}(-3 \zeta)+Q_{1} Q_{2}^{\prime \prime} \eta \zeta+ \\
Q_{1} Q_{2}\left(\frac{\eta^{\prime 2}}{\eta}(3 \zeta)+\eta^{\prime \prime}(-3 \zeta)\right)+ \\
Q_{1}^{\prime} Q_{2}^{\prime} \eta \zeta+Q_{1}^{\prime} Q_{2}^{\prime} \eta^{\prime}(-3 \zeta)
\end{array}\right)
\end{aligned}
$$

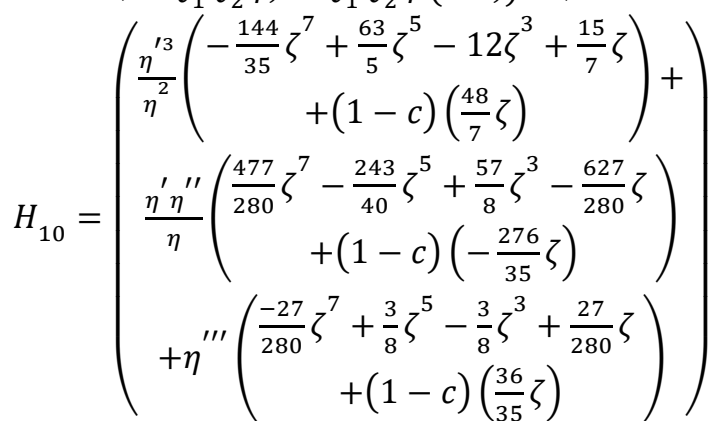

$$
\begin{aligned}
& H_{11}=\left(\begin{array}{c}
\frac{\eta^{\prime 2}}{\eta}\left(\begin{array}{c}
\frac{18}{35} \zeta^{7}-\frac{21}{10} \zeta^{5}+3 \zeta^{3}-\frac{15}{14} \zeta \\
+(1-c)\left(-\frac{24}{7} \zeta\right)
\end{array}\right)+ \\
\eta^{\prime \prime}\left(\begin{array}{c}
\frac{-27}{280} \zeta^{7}+\frac{3}{8} \zeta^{5}-\frac{3}{8} \zeta^{3}+\frac{27}{280} \zeta \\
+(1-c)\left(\frac{36}{35} \zeta\right)
\end{array}\right)+
\end{array}\right) \\
& H_{12}=\left(\begin{array}{c}
\frac{\eta^{\prime 3}}{\eta}\left(\begin{array}{c}
-\frac{3}{2} \zeta^{5}-3 \zeta^{3}+\frac{51}{70} \zeta \\
+(1-c)\left(\frac{132}{35} \zeta\right)
\end{array}\right)+ \\
\eta^{\prime} \eta^{\prime \prime}\left(\begin{array}{c}
3 \zeta^{3}-\frac{51}{35} \zeta \\
+(1-c)\left(-\frac{264}{35} \zeta\right)
\end{array}\right)+ \\
\eta \eta^{\prime \prime \prime}\left(\frac{3}{20} \zeta^{5}-\frac{1}{2} \zeta^{3}+\frac{33}{140} \zeta\right. \\
\left.\left.+(1-c)\left(\frac{18}{35} \zeta\right)\right)\right)
\end{array}\right)
\end{aligned}
$$$$
\begin{aligned}
& H_{13}=\left(\begin{array}{c}
\eta^{\prime 2}\left(\begin{array}{c}
\frac{3}{5} \zeta^{5}+2 \zeta^{3}-\frac{51}{35} \zeta \\
+(1-c)\left(\frac{-264}{35} \zeta\right)
\end{array}\right)+ \\
\eta^{\prime}\left(\begin{array}{c}
\frac{3}{10} \zeta^{5}-\zeta^{3} \frac{33}{70} \zeta \\
+(1-c)\left(\frac{36}{35} \zeta\right)
\end{array}\right)
\end{array}\right) \\
& Q_{1}=\frac{3}{M \eta^{3}}, \\
& Q_{2}=\frac{18 \eta^{\prime} \mu^{\prime}}{M^{2} \eta^{5}}+\frac{27 \eta^{\prime} \mu^{\prime 2}}{M^{3} \eta^{7}} \\
& \zeta=\frac{y}{\eta}
\end{aligned}
$$

\begin{tabular}{|c|c|c|c|}
\hline $\boldsymbol{c}$ & $R e$ & $\gamma$ & $\eta(x)$ \\
\hline 0 & 0.1 & $\begin{array}{c}0.0 \\
\text { (Straight } \\
\text { channel) }\end{array}$ & \multirow{2}{*}{$\begin{array}{c}\text { Stenosis-1 } \\
\eta(x)=0.5\left((2-\gamma)-\gamma \sin \left(2 \pi x-\frac{\pi}{2}\right)\right)\end{array}$} \\
\hline 0.1 & 2 & $\begin{array}{c}0.2 \\
\text { (Mild } \\
\text { stenosis) }\end{array}$ & \\
\hline 0.2 & 4 & $\begin{array}{c}0.4 \\
\text { (Mild } \\
\text { stenosis) }\end{array}$ & $\begin{array}{c}\text { Stenosis-2 } \\
\eta(x)=1+\gamma \sin (\pi x-\pi))\end{array}$ \\
\hline 0.3 & 6 & $\begin{array}{c}0.6 \\
\text { (Moderate } \\
\text { stenosis) }\end{array}$ & Stenosis-3 \\
\hline 0.4 & 8 & $\begin{array}{c}0.8 \\
\text { (Sever } \\
\text { stenosis) } \\
\end{array}$ & $\eta(x)= \begin{cases}1-2 \gamma x & 0 \leq x \leq 0.5 \\
1+2 \gamma(x-1) & 0.5 \leq x \leq 1\end{cases}$ \\
\hline 0.5 & 10 & & \multirow{2}{*}{$\begin{array}{c}\text { Stenosis-4 } \\
\eta(x)=1+4 \gamma\left(x^{2}-x\right)\end{array}$} \\
\hline 0.59 & 12 & & \\
\hline
\end{tabular}

\section{Results and discussion}

The study is made based on different hematocrit $(c)$, different Reynolds number $(R e)$, different area reduction $(\gamma)$ and different geometries of the stenosis $(\eta(x))$ as shown in Table (1).

\section{Table 1-The parameters used in this study}

In perturbation technique, the solution of the problem can be accepted when the values of the higher order can be neglected. The solution of the second order system presented for this model can be sufficient as shown in the Figure (2).

Figure 2 gives the fluid velocity based on the zero, first, and second order respectively, the weight of the third order solution can be neglected because of the solution gives an accepted conversion, specially at the last position shown in Figure (2-c). 




a) Before the minimum area $(x=0.25)$

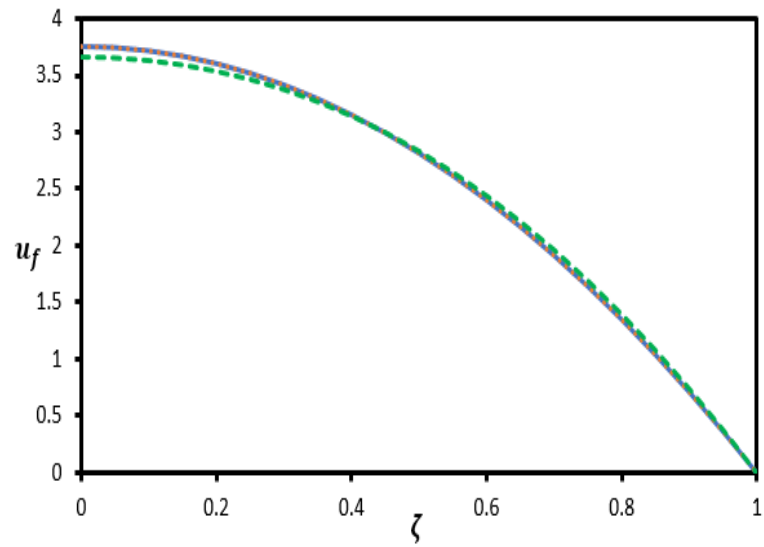

b) At the min area directly $(x=0.5)$

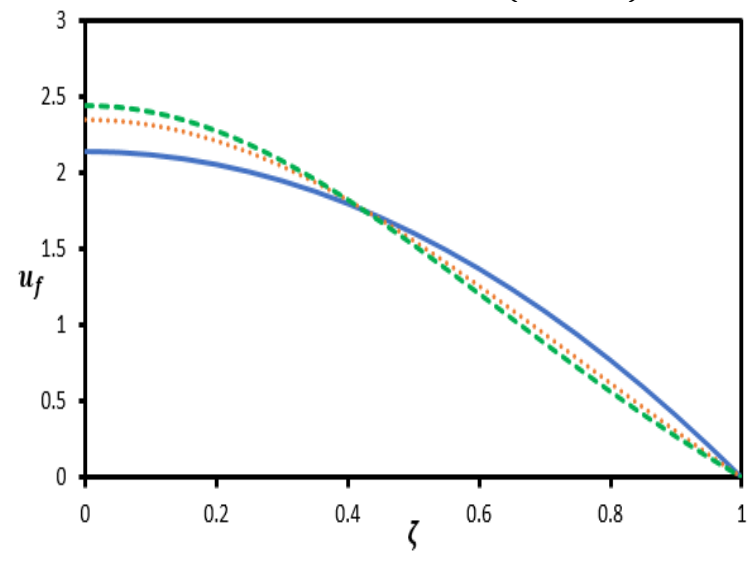

c) After the min area at $(x=0.75)$

Figure 2- Conversion of solution at some positions (c=0.4, $\operatorname{Re}=10$, and $\gamma=0.6)$

Effect of hematocrit change is introduced in Figure (3), as the volume concentration of the red blood cells increase the velocity of the fluid phase decreases at the core region while increases near the walls. This behavior occurs before the stenosis minimum area (in the conversion artery) as shown in Figures. (3-a, 3-b), while this effect reversed after the minimum height (in the divergent artery) as shown in
Figure (3-c).

In the conversion portion, the fluid response to the area change is greater than the particles response, and therefore as the concentration increases the particles number increases causing a declaration to the fluid. While in the divergent portion the momentum transferred to the particles in the previous portion comes back to the fluid causing the fluid to a accelerate. As the hematocrit increases, the momentum transmitted is increasing and the velocity increases as mentioned by El-Askary et al. [30].

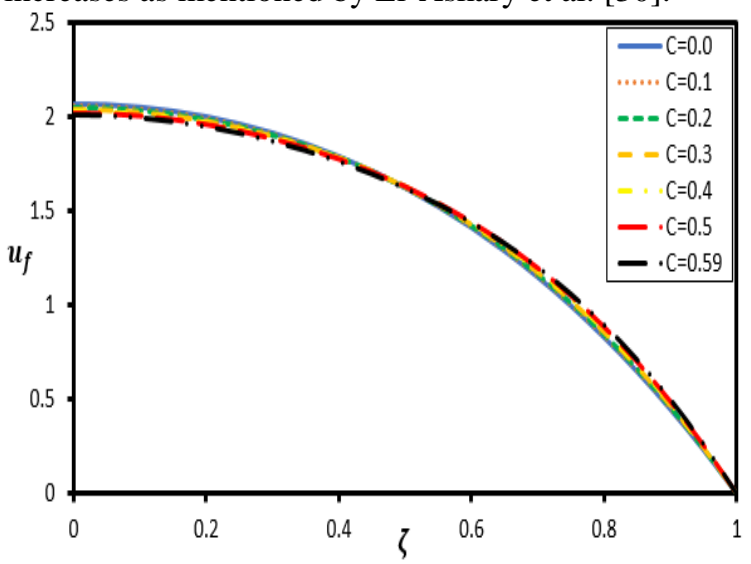

a) Before the minimum area $(x=0.25)$

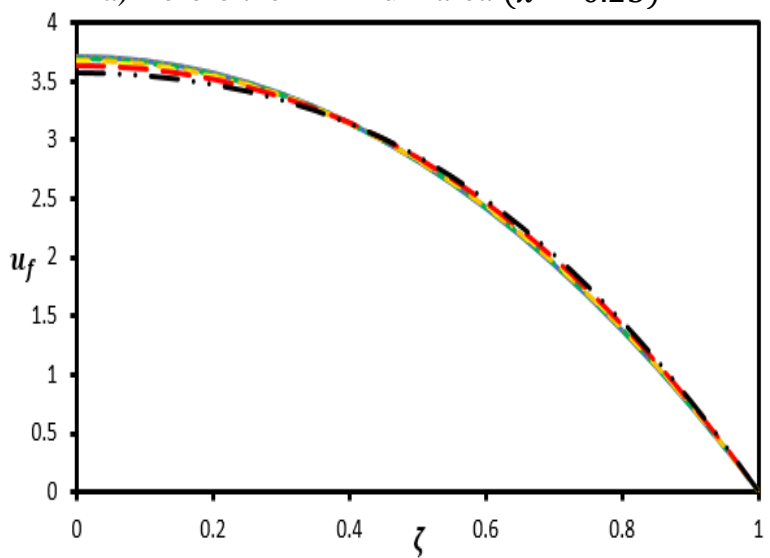

b) At the min area $(x=0.5)$

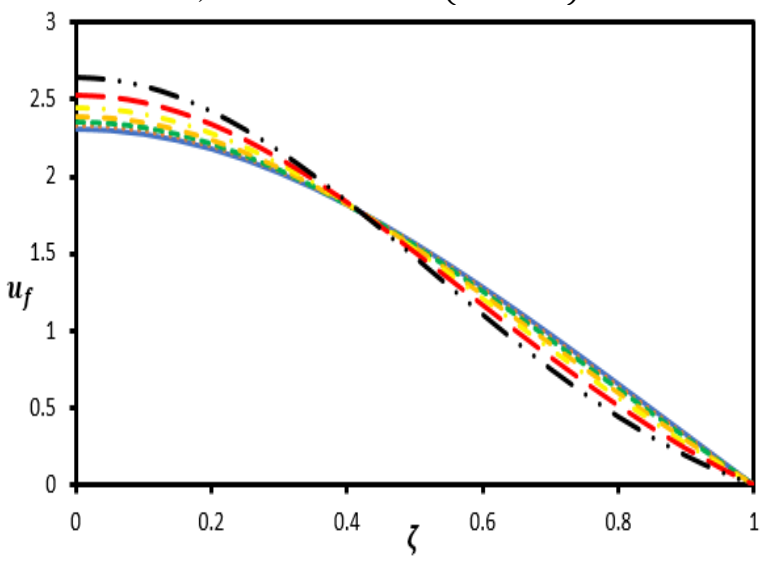

c) After the min area directly $(x=0.75$ 
Figure 3- Effect of hematocrit (c) at different locations at $(\operatorname{Re}=10)$ and $\gamma=0.6$

The fluid velocity behaves under the increase of Reynolds number like its performance in case of hematocrit increases as shown in Figure (4). The fluid velocity can overcome the increase in the Reynolds number than the particles, thus as the Reynolds number increases the momentum transfer increases causing a velocity changes as shown in Figure (4).

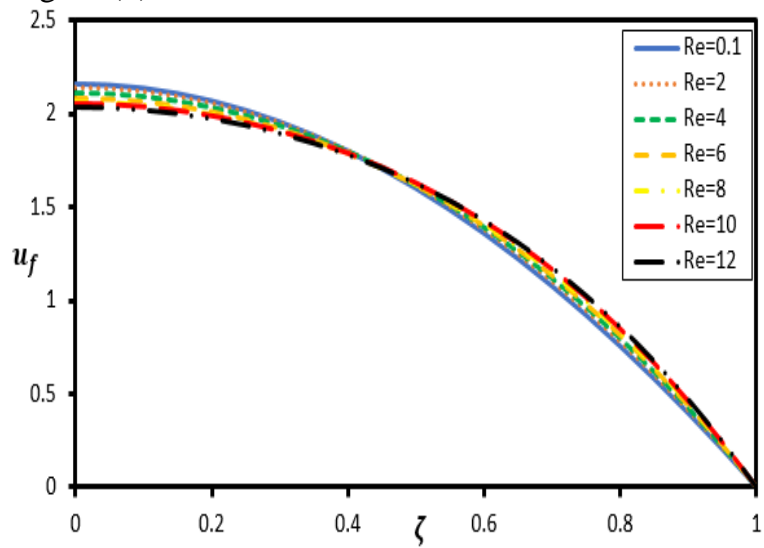

a) Before the minimum area $(x=0.25)$

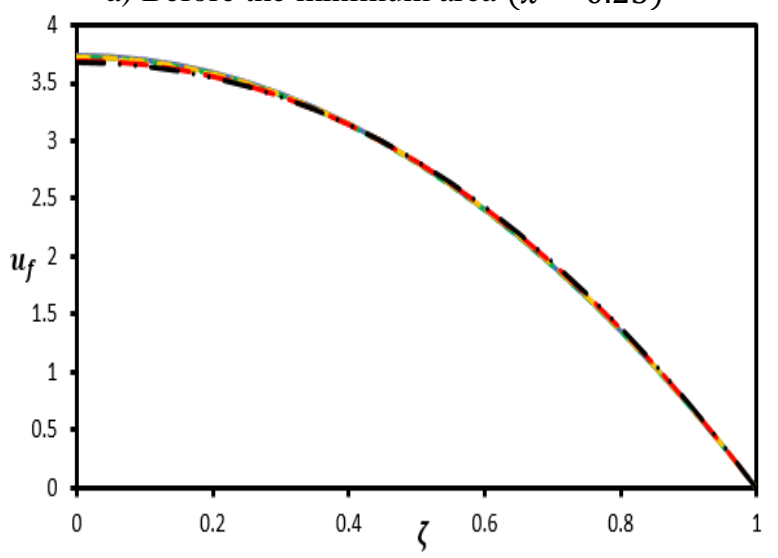

b) At the min area $(x=0.5)$

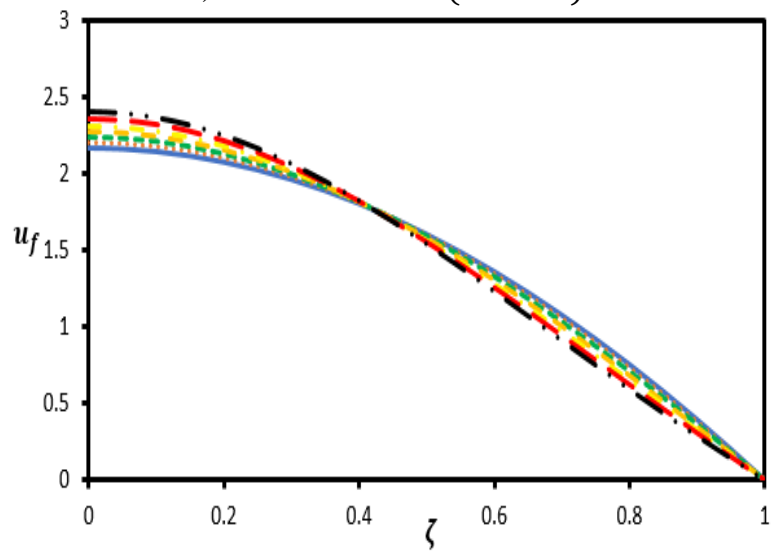

c) After the min area $(x=0.75)$
Figure 4- Effect of Reynold's number (Re) at different locations at ( $\mathrm{c}=0.2$ and $\gamma=0.6)$

Area reduction of the artery affects the fluid velocity as shown in Figure (5). The velocity increases as the area reduction increases, and thus the velocity of the fluid should increase to maintain the flow rate.

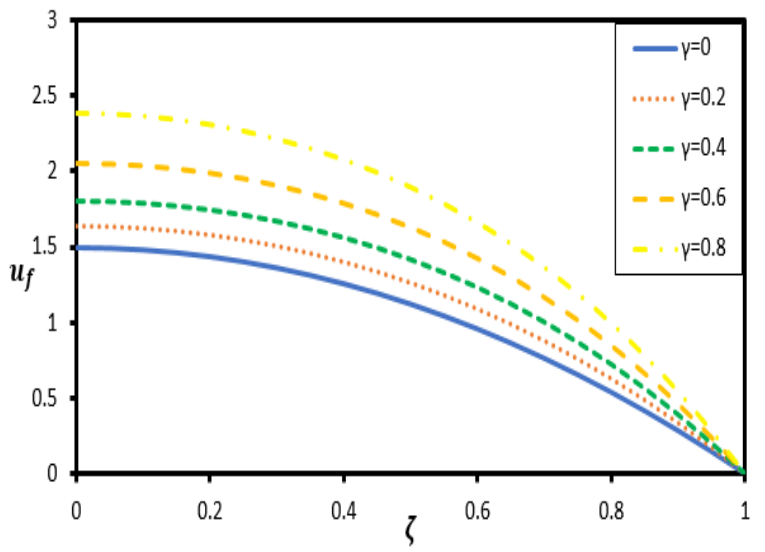

a) Before the minimum area $(x=0.25)$

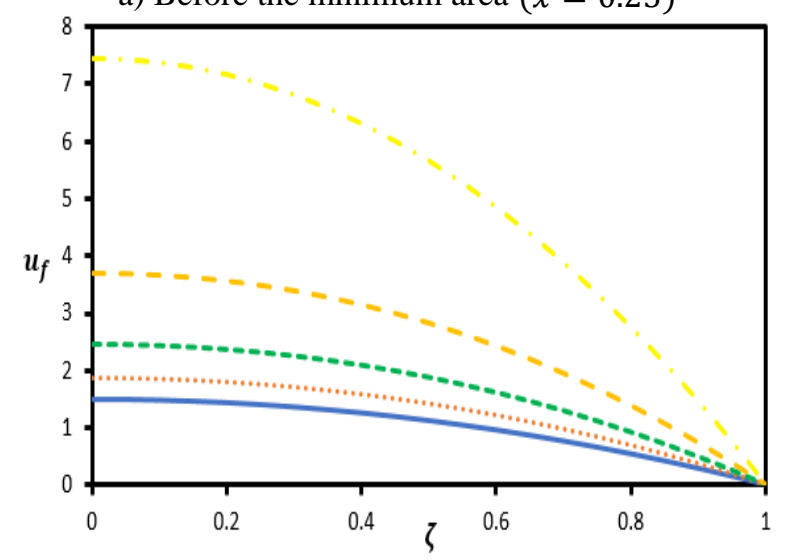

b) At the min area $(x=0.5)$

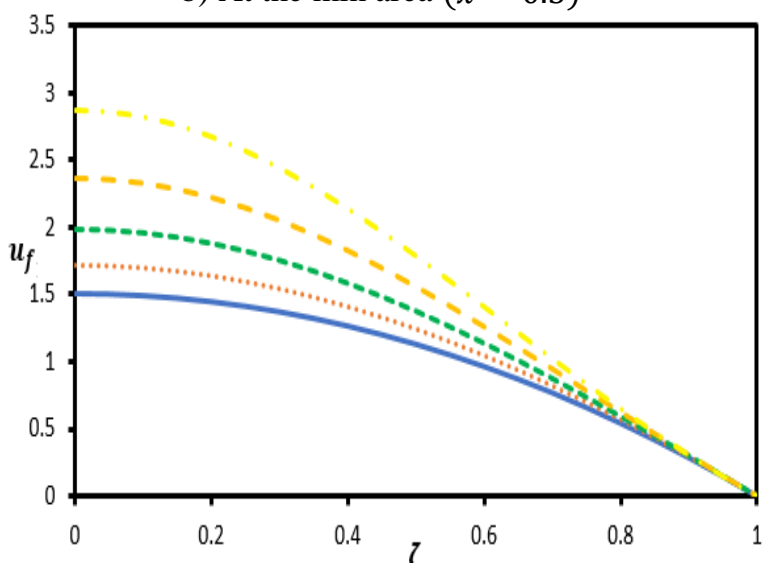

c) After the min area directly $(x=0.75)$

Figure 5- Effect of area reduction $(\gamma)$ at different locations at $(\operatorname{Re}=10$ and $\mathrm{c}=0.2)$ 


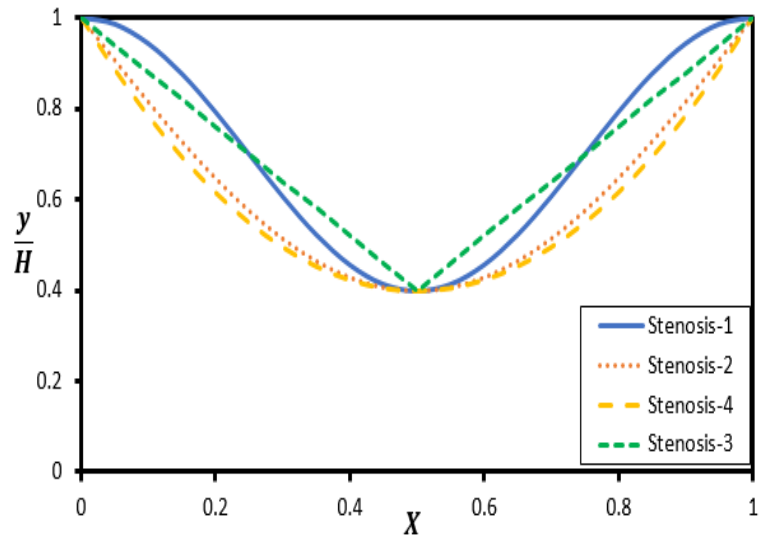

Figure -6 Stenosis wall shape

All four-stenosis mentioned in Table (1) are plotted in Figure (6) with same area reduction $(\gamma=0.6)$ to show the velocity difference in each one as shown in Figure (7). Figure (7-a) shows that the stenosis-1 has the minimum velocity than the other three stenosis at core section, while the straight shaped stenosis (stenosis-3) has a small difference than the first one. Stenosis-4 and stenosis-2 have larger velocity values respectively because of local area at this position.

From Figure (6) the largest narrowing at $\mathrm{x}=0.25$ is Stenosis- 4 and the velocity becomes larger as shown in Figure (5). This effect is also shown in Figure (7c), while Figure (7-b) shows that the effect of the stenosis shape has a noticeable effect (in this section the area reduction is constant). As the change of the slop is greater the velocity at the core is less and vice versa near the walls.

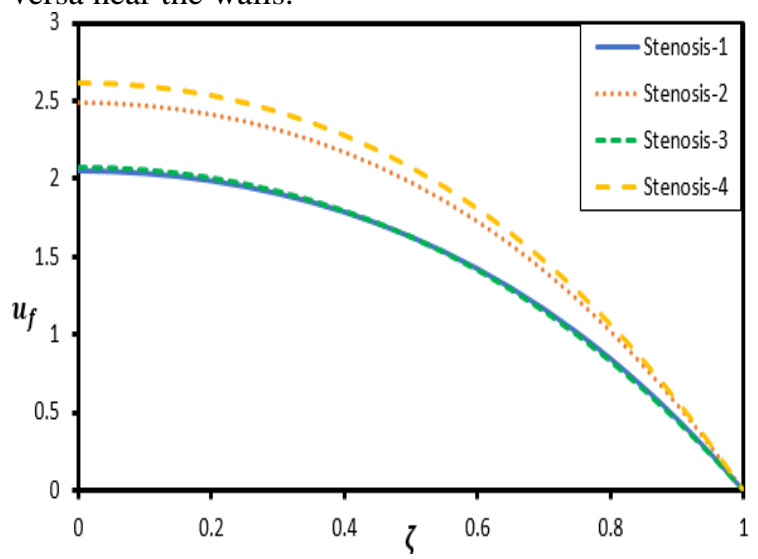

a) Before the min area $(x=0.25)$

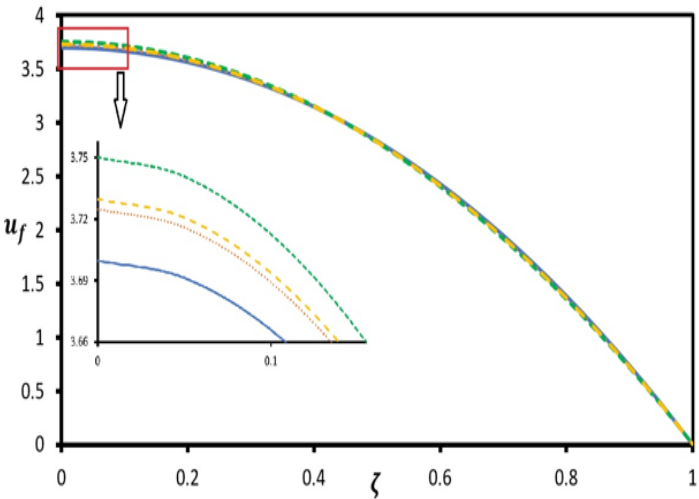

b) At the min area directly $(x=0.5)$

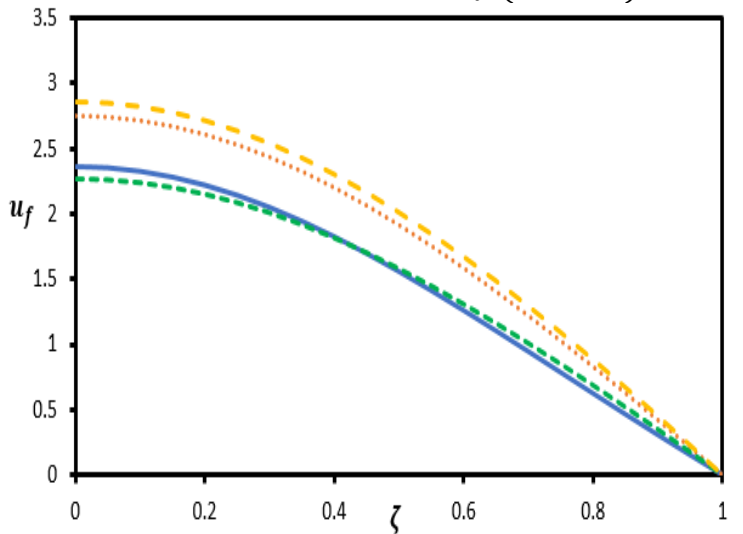

c) After the min area directly $(x=0.75)$

Figure 7- Effect of stenosis geometry at different locations at $(\operatorname{Re}=10, \mathrm{c}=0.2$ and $\gamma=0.6)$

Pressure gradient along axial direction viewed in Figure (8) shows the effect of the hematocrit on the pressure gradient. The value of the pressure gradient decreases with the axial distance till near the minimum height of the stenotic, then increases to its maximum value at distance between 0.68 for higher hematocrit to 0.83 for the single phase, after that it returns to decrease again. The magnitude of the pressure gradient always increases with hematocrit increase.

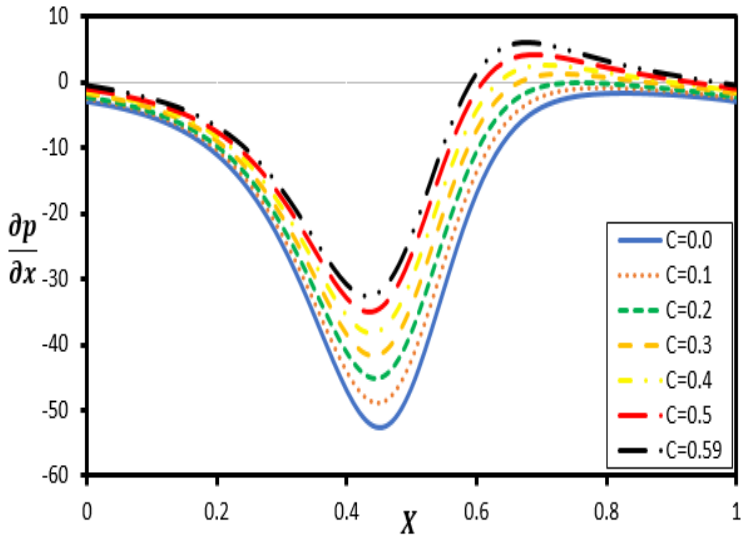

Figure 8- Effect of hematocrit (C) on the pressure gradient at $(\operatorname{Re}=10)$ and $\gamma=0.6$ 
Figure (9) introduces the pressure gradient with the axial distance; as Reynolds number increases the minimum value of the pressure gradient is moving downward the throat and it gives a smaller value until the throat then its value becomes larger than flow with lower Reynolds number.

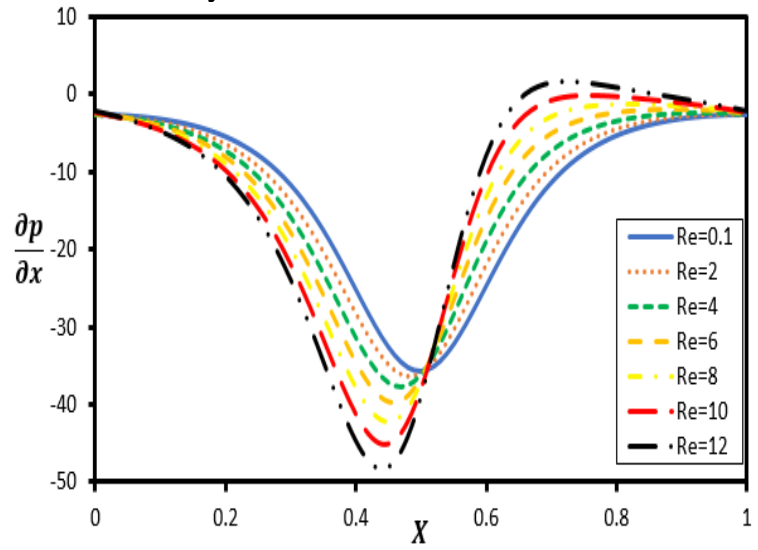

Figure 9- Effect of Reynold's number (Re) on pressure gradient at $(\mathrm{c}=0.2)$ and $\gamma=0.6$

Figure (10) shows the effect of area reduction on the pressure gradient. As the area reduction increases the pressure gradient decreases before the throat. The greater area reduction which is known as a sever stenosis has a pressure gradient 7 times larger than the moderate one (in the absolute value). This great change in the pressure gradient leads to a great change in velocity as shown in Figure (5) leading to a harmful effect as mentioned in [3].

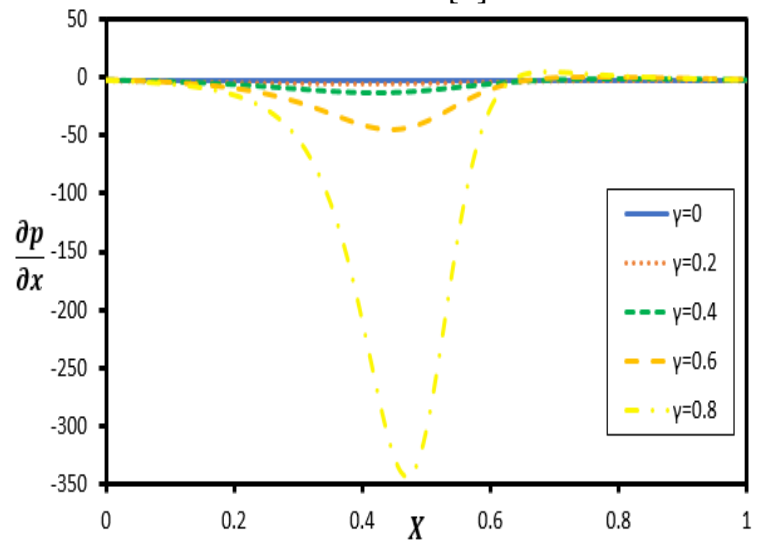

Figure 10- Effect of area reduction $(\gamma)$ on pressure gradient at $(\operatorname{Re}=10)$ and $c=0.2$

The stenotic geometry effects on the pressure gradient are shown in Figure (11). The great change in the walls of the stenotic gives a step change in the pressure gradient as shown by trend of the stenosis-3 curve in Figure (11).



Figure 11- Effect of stenosis geometry on pressure gradient at $(\operatorname{Re}=10), \mathrm{c}=0.2$ and $\gamma=0.6$

The streamlines for different geometry shapes are introduced in Figure (12).

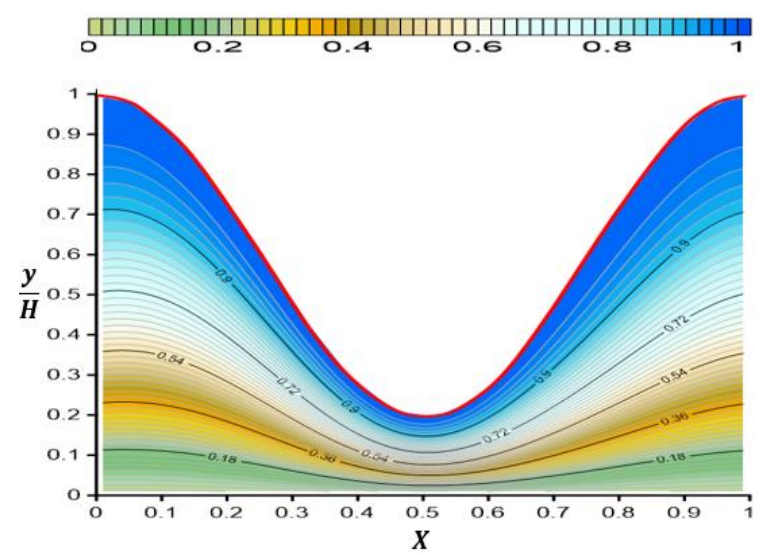

a) Stenosis-1

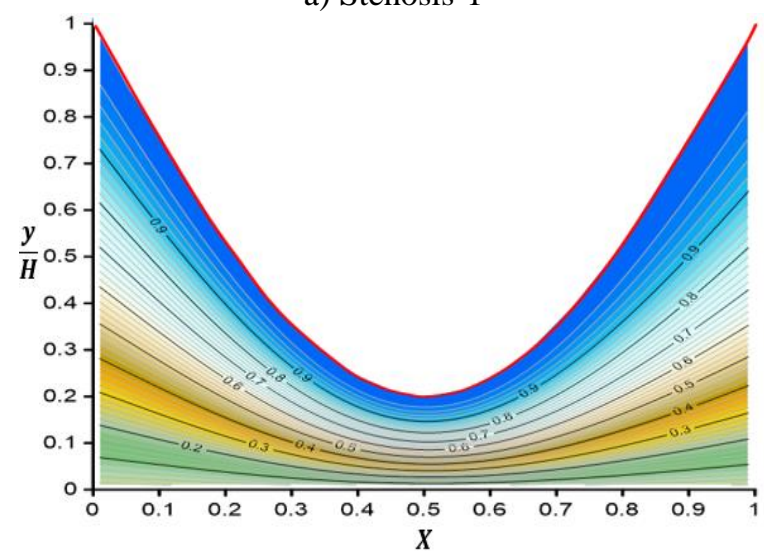

b) Stenosis-2 


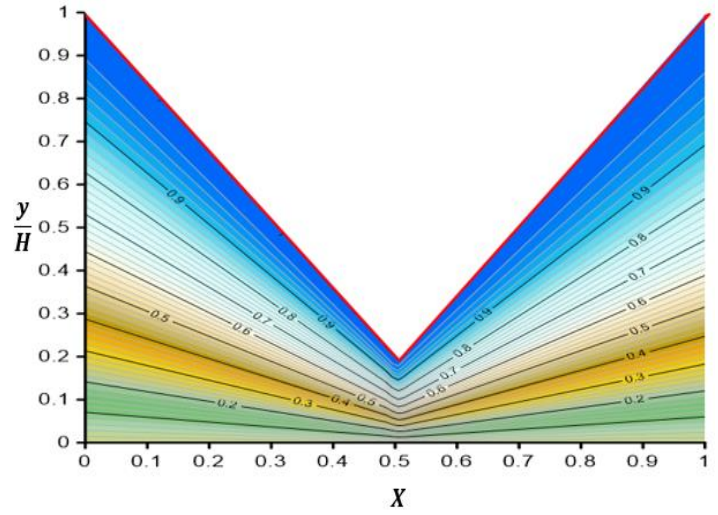

c) Stenosis-3

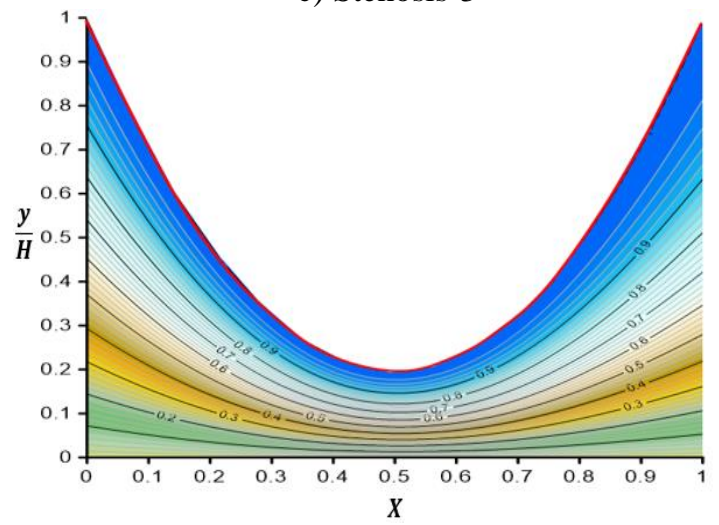

d) Stenosis-4

Figure 12- Effect of different stenosis shape on the stream function

\section{Conclusion}

The geometry of stenosis has a great effect on the pressure gradient in the axial direction, also there is a change in velocity profile in each cross section of the geometry. The hematocrit has a noticed effect on the flow velocity and pressure gradient, as the hematocrit increases the maximum velocity will decrease before the throat while it increases after that section. The effect of Reynolds number will cause a minimum pressure gradient to move downward the stenotic height. The increase in Reynolds number affects the maximum velocity as the hematocrit effect.

\section{References}

[1] N. Alexopoulos, D. Katritsis and P. Raggi, "Visceral adipose tissue as a source of inflammation and promoter of atherosclerosis," Atherosclerosis, vol. 233, pp. 104-112, 2014.

[2] B. Tripathi, B. K. Sharma and M. Sharma, "MHD Pulsatile Two-Phase Blood Flow Through a Stenosed Artery with Heat and Mass Transfer," arXiv preprint arXiv:1705.09794, 2017.
[3] H. Huang, V. J. Modi and B. R. Seymour, " Fluid mechanics of stenosed arteries.," International Journal of Engineering Science., vol. 33, pp. 815-828, 1995.

[4] I. M. Eldesoky, "Unsteady MHD pulsatile blood flow through porous medium in a stenotic channel with slip at the permeable walls subjected to time dependent velocity (injection/suction)," The International Conference on Mathematics and Engineering Physics, vol. 7, pp. 1-25, 2014, May.

[5] S. Kumar, S. Kumar and D. Kumar, "Oscillatory mhd flow of blood through an artery with mild stenosis," INTERNATIONAL JOURNAL OF ENGINEERING, vol. 22, pp. 125-130, 2009.

[6] S. Mishra, S. U. Siddiqui and A. Medhavi, "Blood flow through a composite stenosis in an artery with permeable wall.," An International Journal, vol. 6, pp. 58-73, June 2011.

[7] A. Rohela and J. S. Chaudhary, "Mathematical Study of Effect of Magnetic Field on Blood Flow in Stenotic Artery," International Journal of Engineering and Mathematical Sciences, vol. 6, pp. 16-22, 2014.

[8] S. Kumar and C. Diwakar, "A mathematical model of power law fluid with an application of blood flow through an artery with stenosis," Applied Mathematically Bio-Sciences, vol. 4, pp. 25-36, 2013.

[9] R. Bali and U. Awasthi, "Effect of a magnetic field on the resistance to blood flow through stenotic artery.," Applied Mathematics and Computation., vol. 188, pp. 1635-1641, 2007.

[10] R. R. Ellahi, S. U. Rahman, S. Nadeem and K. Vafai, "The blood flow of Prandtl fluid through a tapered stenosed arteries in permeable walls with magnetic field.," Communications in Theoretical Physics., vol. 63, pp. 353-358, 2015.

[11] I. . A. Mirza, M. Abdulhameed and S. Shafie, "Magnetohydrodynamic approach of nonNewtonian blood flowwith magnetic particles in stenosed artery.," Applied Mathematics and Mechanics., vol. 38, pp. 379-392, 2017.

[12] D. F. Young, " Effect of a time-dependent stenosis on flow through a tube.," Journal of Engineering for Industry., vol. 90, pp. 248-254, 1968.

[13] M. M. Bhatti , M. Marin, A. Zeeshan, R. Ellahi and S. I. Abdelsalam, "Swimming of Motile Gyrotactic Microorganisms and Nanoparticles in Blood Flow Through Anisotropically Tapered Arteries," Frontiers in Physics, vol. 8, p. 95, 2020. 
[14] S. A. Berger and L. D. Jou , "Flows in stenotic vessels. Annual review of fluid mechanics," Annual Review of Fluid Mechanics, vol. 32, pp. 347-382, 2000.

[15] J. C. Chow and K. Soda, "Laminar flow in tubes with constriction.," The Physics of Fluids., vol. 15, pp. 1700-1706., 1972.

[16] J. F. Chow and K. Soda, "Laminar flow and blood oxygenation in channels with boundary irregularities.," Journal of Applied Mechanics., vol. 40, pp. 843-850, 1973.

[17] D. S. Sankar and U. Lee, "Two-phase non-linear model for the flow through stenosed blood vessels.," Journal of Mechanical Science and Technology., vol. 21, pp. 678-689, 2007.

[18] D. S. Sankar and U. Lee, "Mathematical modeling of pulsatile flow of non-Newtonian fluid in stenosed arteries.," Communications in Nonlinear Science and Numerical Simulation., vol. 14, pp. 2971-2981, 2009.

[19] D. . S. Sankar and U. Lee, "Two-fluid Casson model for pulsatile blood flow through stenosed arteries: A theoretical model.," Communications in Nonlinear Science and Numerical Simulation., vol. 15, pp. 2086-2097, 2010.

[20] D. C. Sanyal and B. Sarkar, "Analysis of wavy flow and blood oxygenation in two-dimensional channels with constriction.," Journal of Applied Mathematics and Computing., vol. 26, pp. $397-$ 417, 2008.

[21] V. P. Srivastava, "Two-phase model of blood flow through stenosed tubes in the presence of a peripheral layer: applications.," Journal of Biomechanics., vol. 26, pp. 1377-1382., 1996.

[22] V. P. Srivastava, "Particulate suspension blood flow through stenotic arteries: Effects of hematocrit and stenosis shape.," Indian Journal of Pure and Applied Mathematics., vol. 33, pp. 1353-1360., 2002.

[23] V. P. Srivastava and R. Rastogi, "Blood flow through a stenosed catheterized artery: Effects of hematocrit and stenosis shape.," Computers \& mathematics with applications., vol. 59, pp. 1377-1385., 2010.

[24] B. E. Morgan and D. F. Young, " An intergral method for the analysis of flow in arterial stenoses.," Bulletin of Mathematical Biology., vol. 36, pp. 39-53., 1974.

[25] U. S. Chakraborty, D. Biswas and M. Paul, "Suspension model blood flow through an inclined tube with an axially non-symmetrical stenosis," Korea-Australia Rheology Journal, vol. 23, pp. 25-32, 2011.
[26] G. R. Charya, "Pulsatile flow of a dusty fluid through a constricted channel," Journal of Applied Mathematics and Physics, vol. 29, pp. 217-225, 1978.

[27] A. R. Sankar, S. R. Gunakala and D. M. Comissiong, "Two-layered blood flow through a composite stenosis in the presence of a magnetic field," International Journal of Application or Innovation in Engineering and Management, vol. 2, pp. 30-41, 2013.

[28] S. I. Abdelsalam and K. Vafai, "Particulate suspension effect on peristaltically induced unsteady pulsatile flow in a narrow artery: blood flow model," Mathematical biosciences, vol. 283, pp. 91-105, 2017.

[29] I. M. Eldesoky, S. I. Abdelsalam, W. A. ElAskary, A. M. El-Refaey and M. M. Ahmed, "Joint effect of magnetic field and heat transfer on particulate fluid suspension in a catheterized wavy tube," BioNanoScience, vol. 9, pp. 723739, 2019.

[30] W. A. El-Askary, I. M. Eldesoky, O. Saleh, S. M. El-Behery and A. S. Dawood, "Behavior of downward turbulent gas-solid flow through sudden expansion pipe," Powder Technology, vol. 291, pp. 351-365, 2016. 